\title{
An Efficient Computer-Aided Diagnosis System for the Analysis of DICOM Volumetric Images
}

\author{
QOSEEN ZAHRA*, MUHAMMAD SHERAZ ARSHAD MALIK*, AND NAILA BATOOL* \\ RECEIVED ON 19.06.2018 ACCEPTED ON 30.10.2018 \\ ABSTRACT
}

\begin{abstract}
Medical images are an important source of diagnosis. The brain of human analysis is now an advanced field of research for computer scientists and biomedical physicians. Services provided by the healthcare units usually vary, the quality of treatment provided in the urban and rural generally not same. Unavailability of medical equipment and services can have serious consequences in patient disease diagnosis and treatment. In this context, we developed. MRI (Magnetic Resonance Imaging) based CAD (Computer Aided Diagnosis) system which takes MRI as input and detects abnormal tissues (Tumors). MRI is the safe and well reputed imaging methodology for prediction of tumors. MRI modality assists the medical team in diagnosis and proper treatment plan (Medication/Surgery) of different types of abnormalities in the soft tissues of the human body. This paper proposes a framework for brain cancer detection and classification. The tumor is segmented using a semi-automatic segmentation algorithm in which the threshold values selection for head and cancer regions are premeditated automatically. Segmented tumors are further sectioned into malignant and benign using SVM (Support Vector Machine) classifier. Detailed experimental work indicates that our proposed CAD system achieves higher accuracy for the analysis of brain MRI analysis.
\end{abstract}

Key Words: Magnetic Resonance Imaging, Support Vector Machine Classifier, Bags of Visual Words, Computer Aided Diagnosis System, Digital Imaging and Communications in Medicine Volumetric Images, 2D and 3D Images.

\section{INTRODUCTION}

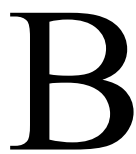

io-Health imaging is the strongest part of the study during the past few years donating to culture in several behaviors. Several image methodologies are used for the health field, like as MRI, endoscopy, CT (Computerized Tomography) scan, etc. These images methodologies perform a vast role in present health measures for research education, diagnosis, analysis, and dealing with multi-factorial health sicknesses. The health images play a central role in the act of any radiology section. Health descriptions need processing of image techniques in computer vision, such as development and segmentation of an image [1] to find-out analytic figures like recognition of damaging area or disease. The brain of human analysis is curretly an advanced research field for computer scientists and bio-medical physicians [2]. The brain is the core part of

Authors E-Mail: (qoseenzahra@gmail.com, sheraz_awan@gcuf.edu.pk, nailabatool93@gmail.com)

* Department of Information Technology, Government College University, Faisalabad, Pakistan.

This is an open access article published by Mehran University Research Journal of Engineering and Technology, Jamshoro under the CC by 4.0 International License. 
the human CNS (Central Nervous System), it is protected via a strong bone called a skull. The total average mass of a normal human brain is $1300-1500 \mathrm{mg}$ and volume is $1260 \mathrm{~cm}^{3}$. The brain consists of around 86 billion neurons (perceptron), glial cells and vessels. It can be classified into fluid, GM (Gray Matter) and WM (White Matter). GM may easily be distinguished from the white matter due to its color. Spinal and brain cord are bounded by a liquid called CSF (Cerebrospinal Fluid). Thus in a natural system in new cells are born and the old cells are replaced, but in case of tumor the old cell does not die and grows which can be very dangerous for human life. Broadly there are two of tumors such as, malignant and benign. In case of the benign cancer, evolution is slow and cells are smaller in size. If does not affect the normal cells and spread. However, the malignant growths are a cancerous lump, larger in size, and growth of cells are faster.

MRI is the secure and famous imaging methodology for tumor prediction in radiology. It creates 3D (ThreeDimensional) DICOM (Digital Imaging and Communications in Medicine) volumetric images of the brain. MRI technologies produce volumetric descriptions and support the radiologists to research on the human brain in depth. Physical analysis of skull MRI is time taking and vulnerable to errors due to the size of MRI data and complexity for every patient. So, many state-of-art segmentation methods have been presented for this type of situation; each technique has both advantages and disadvantages [3]. Most of the work done on brain MRI analysis has been made on a single 2D image, which has been converted from DICOM format to simple image format (.png, .jpg etc). The segmentation of brain and tumor in a single image is easier than the segmentation of volumetric images, but the results obtained are not beneficial for the surgeons and radiologists. The segmentation phase is also an important phase for several dimensions such as calculation of tumor volume in cubic millimeter, 3D therapy $360^{\circ}$ rotatation, and sorting of cancer type into benign and malignant. Thresholding typed segmentation is a famous technique for the MRI volumetric image segmentation [4]. Working of threshold-type segmentation is depended on the collection of a preferable value of the threshold.

Physical collection for the correct edges is complicated and needs machine learning knowledge. For this kind of operational test support unitbinarized auto-segmented brain and tumor parts with the support of selecting threshold correct values [5]. This Otsu binarized way is cheap to apply but it is noisaters and sustains segmenting cancer having similar existence to other brain portions. In the Watershed [6] segmentation, objects are overlapping because it is a robust technique for segmentation. Watershed applies with the magnitude of gradient on the image to find segment objects of interest or tumor. The overall performance of watershed technique is good, but it is not satisfactory for 3D volumetric classification. Clustering is an unverified knowledge method in the brain knowledge used to combine unbalanced items with matching characteristics in a collection [7]. According to clustering scenario procedures, K-map is recognized as a clustering procedure that is utilized for image processing and collecting based facts [8]. Area increasing [9] known as segmentation technique of image and mounted on optimal seed facts, region growing algorithm can be single-seeded or multi-seeded. Multi-seeded point region growing is better than a single-seeded point because the region grows algorithm is very sensitive to noise. Area increasing algorithm is a partial automatized technique because the seed points are selected manually, so choosing a good kernel edge is a complicated job. FCM (Fuzzy C Mean) [10] is a vast familiar collecting procedure same as K-map and other FCM conversion is more complicated than K-map image division [11]. 
In the current research, a hybrid segmentation algorithm is developed. A GUI (Graphical User Interface) component is used to select five seeds from the brain and tumor region. Based on these five seeds automatic threshold values for brain and tumor are calculated. After image-segmenting the human brain into various neurons, classification is applied to each tissue to analyze whether parts are damaged or not. In this situation, the reason for the classification of tumors is to create an effective and robust machine to predict the type of tumor. BOVW (Bag of Visual Words) features extraction method is a robust method for the removal from the image in the computer vision system. This was adopted for the arrangement of infected point class and typically does not provide a reasonable response for the textures holding spatial revolution in large volume, for example, translation and rotation. MRI consist of a geometrical change in large volume. In the planned work, BOVW is enlarged by SURF (Speeded Up Robust Features) structures for real categorization of brain cancer for drilling metaphors of malignant and benign cancers were found beside their crushing reality. After, classification and segmentation, brain size can be designed by using the material found from DICOM and tumor region.

\section{LITERATURE REVIEW}

In this review, both sgmentation and classification are discussed. Image segmentation is the pre-processing operations in a medical image that is done before classification and other post-processing operations including volume calculation and $3 \mathrm{D}$ visualization.

Natarajan et. al. [4] represented a very basic and useful structure for the improvement and the segmentation of the magnetic resonance. In the data sharing phase, the image is reinforced by the equalization of the histograms and the morphological operations are applied to eliminate unwanted points. A middling filter is functional for the elimination of salt and pepper noise from the images. Lastly, the threshold-based guideline segmentation method is used to perceive the tumor. Threshold segmentation offers improved outcomes than programmed and semi-programmed segmentation procedures, though, it is affluent because of the manual assortment of a suitable threshold.

Zhang et. al. [1] represented a brief study on present methods associated with MRI brain study with deliberating many partition procedures for brain cancer segmentation [17]. Several edges-based segmentation approaches fluctuating from physical to involuntary edging have been discussed. Every approach has its own benefits and drawbacks. A further illustration, Otsuthresholding is also an image division procedure which mechanically picks an optimum threshold value with diverse statistical procedures between forefront voxels and background voxels. Region mounting is an other familiar segmentation procedure grounded on solitary seed or multi-seed point assortment. The evolution starts from the seed point and a homogeneousness standard is followed grounded on voxels strengths values. This method delivers good results, but it is very subtle to difference variation, noise and the assortment of best seed point is a challenging job.

Khotanlou et. al. [10] proposed unsupervised fuzzy data grouping algorithm for the segmenting MRI volumetric facts, i.e. K-map. Fuzzy separation procedure splits the voxels in two or more added modules integrating a fixed number of uncertain sets for the individual category. Fuzzy clustering-based segmentation procedure has more complication in stretch time than K-map. 
Deng et. al. [9] offered cancer division structure that practiced personalized area mounting procedure with boundaries and statistical constraints, overwhelming the encounters of physical thresholding. In this technique, the gradient data is retained, so the average variance of the edge curve is designed. In the opinion of authors, improved segmentation results were obtained by means of this method.

Yazdani et. al. [12] offered a well-organized and programmed technique that was more appropriate and precise for the segmentation of GM, WM, and CSF from MRI volumetric images. This technique involved three steps: segmentation was achieved by histogram-based segmentation technique, then texture structures of the segmented regions were mined, and lastly, SVM-classifier was utilized for the arrangement.

Galen et. al. [16] proposed a completely involuntary brain cancer division framework for 3D MRI data. They, initials regularize Gaussian combination technique to the classical standard brain and then a 2D FVF (Fluid Vector Flow) procedure is protracted to $3 \mathrm{D} \mathrm{FVF}$. This technique is verified on a limited dataset with for brain cancer division.

In Albarracin. et al. [14], a statistically based method i.e. tissue possibility maps is a programmed. Segmentation technique proposed by the author identifies the tumor type and predicats class for the tumor. The offered work is verified on two typical brain cancer datasets (i.e. BRATS 2013 and Leaderboard ${ }^{1}$ ).

In Zakeri et. al. [15], brain tumors are satisfied by mining shape based structures (that is, peculiarity, solidness, alteration region-hull-rectangular, variance region-massrectangular, cross-correlation left-hand, and crosscorrelation right-hand) and consistency structures of the extract ROI (Region of Interest). An SVM classifier is trained by the extracted shape and texture structures from the exercise images, while in the testing stage and a class tag is predicated to the innovative challenging facts. Authors clamed $95 \%$ precision.

Galen et. al. [16] offered an involuntary classification technique i.e. wavelet-energy based method for the arrangement of MRI volumetric brain metaphors into usual or irregular. The results of BBO-KSVM were better than BP-NN (Back Propagation Neural Network), KSVM (Kernel Support Vector Machine), and PSO (Particle Swarm Optimization)

\section{METHODOLOGY}

The proposed framework involves two stages:

(1) Calculation of an optimum threshold for segmentation of brain and cancer sections

(2) Classification using machine learning algorithm of the subdivided ROI into the malignant or benign class using SVM classifier. Structures extraction is done using SURF created BOVW.

For vigorous subdivision, a semi-automated threshold collection method is proposed, the projected semiautomatic technique is very fit for the segmentation of MRI volumetric images. BOVW is assumed for the variety and extraction of concerned points in an image using SURF descriptor. The overall sight of the planned framework is shown in Fig. 1.

\subsection{Brain Tumor Segmentation from Volumetric MRI Using Global Thresholding Algorithm}

MRI is processed for the sake of enhancement, restoration, and extraction of expressive data from the images. MRI uses the 16-bit monochrome DICOM format 
which is indeed complex and time consuming for processing manually and automatically. Similarly, the brain MRI data not only consists of the brain, but it also contains skull, Cerebrospinal fluid, skin, and fats. Threshold segmentation is easy and more commonly used in CV (Computer Vision) and image processing application. The aim of thresholding is to divide a copy into two clusters: one cluster will be the foreground and the other cluster will have background values. The single and Multilevel threshold value is a manual technique and a threshold value calculation for single image 2D MRI slices does not provide satisfactory and meaningful segmentation results. A better approach is to segment $3 \mathrm{D}$ volumetric data automatically.

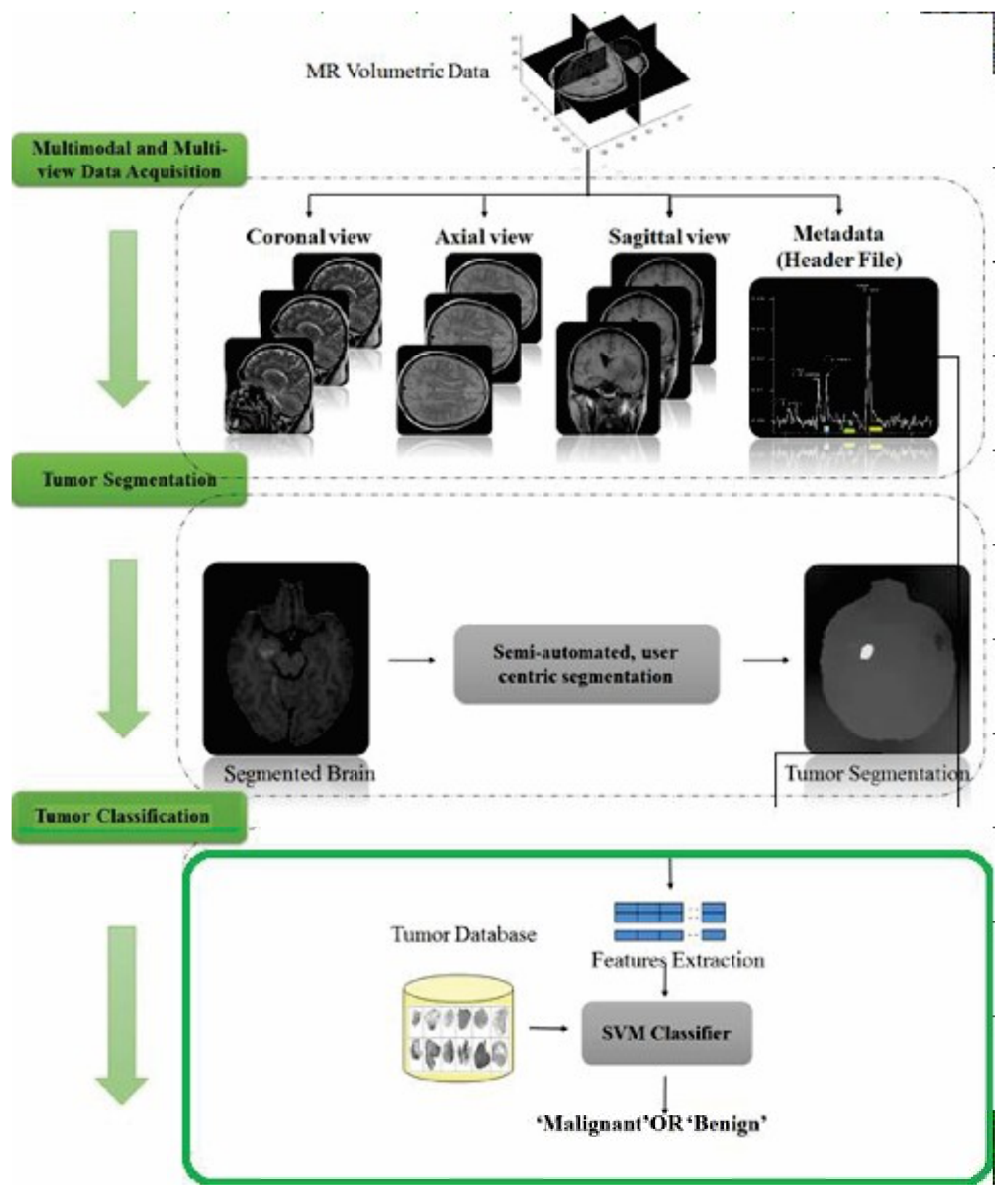

FIG. 1. FRAMEWORK FOR BRAIN TUMOR SEGMENTATION AND CLASSIFICATION [1]

Mehran University Research Journal of Engineering \& Technology, Volume 38, No. 3, July, 2019 [p-ISSN: 0254-7821, e-ISSN: 2413-7219] 
Otsu based automatic segmentation is used in $\mathrm{CV}$ and image processing to cluster an image in bi-levels or to reduce the gray levels 0-65536 into binary $0-1,0$ represent the background and 1 represent the foreground. This method creates a bi-modal histogram separating the gray levels into two separate classes for the sake of finding an optimal threshold value, the combined spread (intraclass variance) should be minimal. Otsu segmentation computes an optimal threshold value using different statistical parameters (weights, standard deviation, and variance) for the segmentation of interest regions in an image. The voxels are clustered on the basis of optimal threshold value, intensities greater than or equal to the optimal threshold are clustered as foreground voxels and intensities less then optimal threshold are clustered as background voxels.

The algorithm of OTSU is explained using a 6x6 image with only 6 grayscale levels as shown in Fig. 2(a-b).

\subsection{Calculation for an Optimal Threshold Value}

Calculating the foreground and background variance for obtaining an optimal threshold value is show in Fig. 3. Following steps are explained in [21].

1. Weight of Background Voxels:

$\mathrm{W}_{\mathrm{b}}=(8+7+2) / 36=0.4722$

2. Mean of Background Voxels:

$\mu_{\mathrm{b}}=((0 \times 8)+(1 \times 7)+(2 \times 2)) / 17=0.6471$

3. Variance of Background Voxels:

$\sigma_{\mathrm{b}}^{2}=\left(\left((0-0.6471)^{2} \mathrm{x} 8\right)+\left((1-0.6471)^{2} \mathrm{x} 7\right)+\right.$

$\left.\left((2-0.6471)^{2} \mathrm{x} 2\right)\right) / 17=0.4637$

4. Weight of foreground Voxels:

$\mathrm{W}_{\mathrm{f}}=(6+9+4) / 36=0.5278$
5. Mean of foreground Voxels: $M_{f}=((3 \times 6)+(4 \times 9)+(5 \times 4)) / 19=3.8947$

6. Variance of foreground Voxels: $\sigma_{\mathrm{f}}^{2}=\left(\left((3-3.8947)^{2} \mathrm{x} 6\right)+\left((4-3.8947)^{2} \mathrm{x} 9\right)+\right.$ $\left.\left((53.8947)^{2} \mathrm{x} 4\right)\right) / 19=0.5152$

7. Calculating the Intra Class Variance:

$$
\begin{aligned}
& \sigma^{2}{ }_{w}=W_{b} \sigma_{b}^{2}+W_{f} \sigma^{2}{ }_{f} \\
& \sigma^{2}{ }_{w}=0.4722 * 0.4637+0.5278 * 0.5152 \\
& \sigma^{2}{ }_{w}=0.4909
\end{aligned}
$$

MRI volumetric data is represented using the histogram in Fig. 4. Automatic segmentation of brain and tumor regions is shown in Fig. 5.

Morphological operations are performed on the segmented brain and tumor images to remove unwanted areas/voxels from the segmented brain and tumor images. In these operations, a $2 \mathrm{D}$ or $3 \mathrm{D}$ small image (kernel) is created which consists of dissimilar kinds of numerical

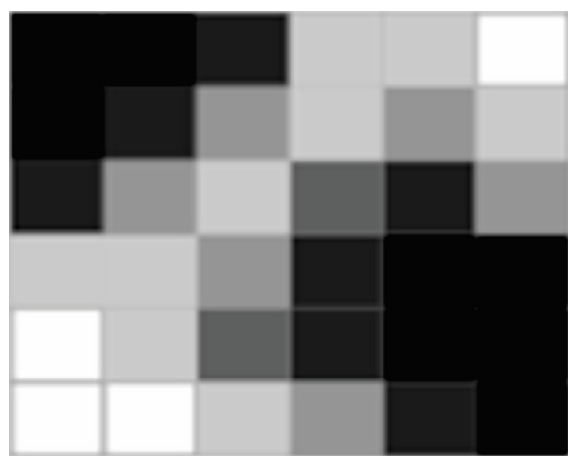

FIG. 2(a). GRAYSCALE IMAGE

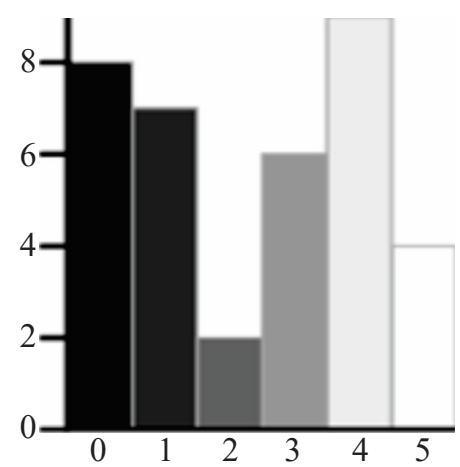

FIG. 2(b). INTENSITY DISTRIBUTION ON HISTOGRAM 
values depending on the type of operation to be performed on the segmented images. 2D kernels perform erosion/dilation in different angles i.e. Horizontal, Vertical,
Diagonally and non-directional. Similarly, the 3D kernel performs the same as $2 \mathrm{D}$ but an extra $3 \mathrm{D}$ for the number of slices in the MRI volumetric images is added. The working

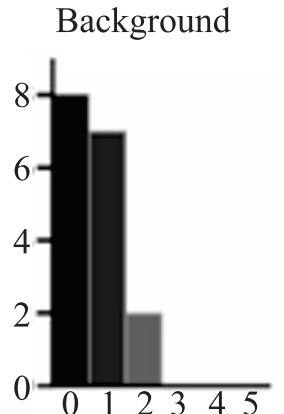

8 pixels

7 pixels 2 pixels
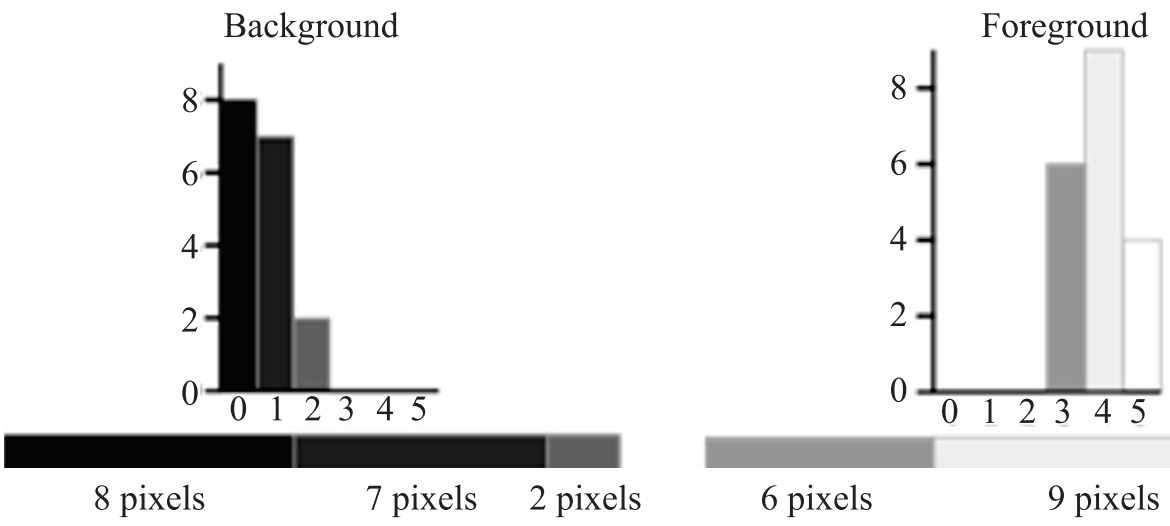

FIG 3. SEPARATING THE BACKGROUND AND FOREGROUND VOXELS

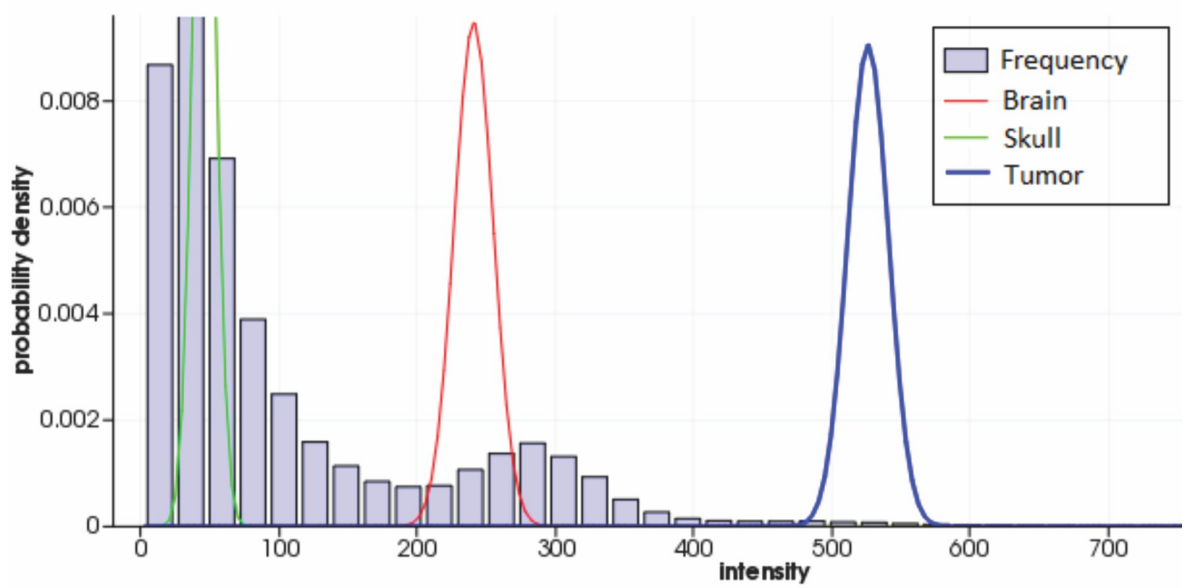

FIG. 4. HISTOGRAM OF MRI VOLUMETRIC DATA
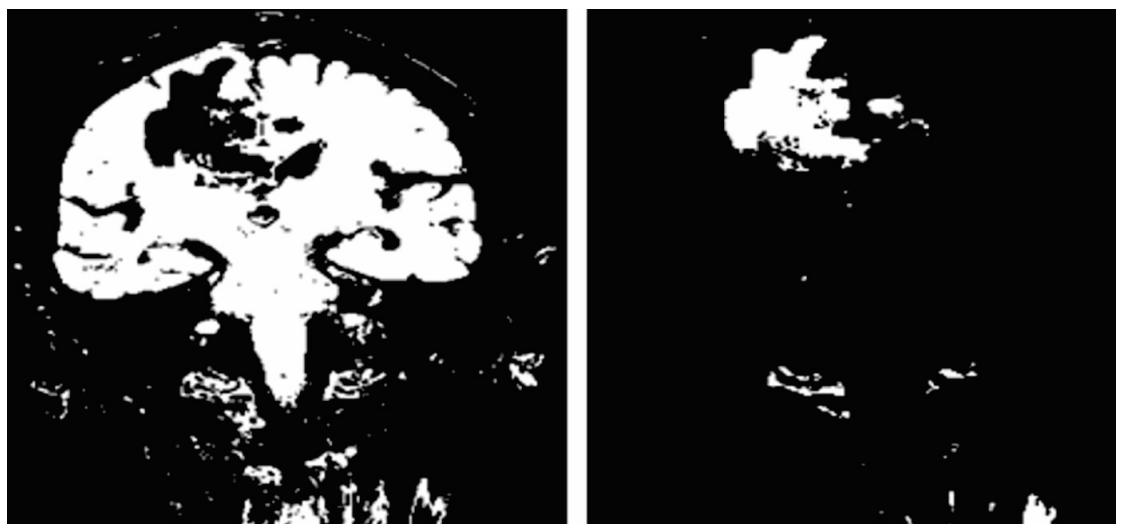

FIG. 5. AUTOMATIC SEGMENTATION OF BRAIN AND TUMOR REGIONS

Mehran University Research Journal of Engineering \& Technology, Volume 38, No. 3, July, 2019 [p-ISSN: 0254-7821, e-ISSN: 2413-7219] 
mechanism of morphological erosion and opening is almost the same but in the situation of volumetric images, morphological erosion performs better. All the proposed work is shown in Figs. 6(a-b)-7(a-b).

\subsection{Features Extraction using Edge Histogram Descriptor}

In our proposed framework, we used EHD (Edge Histogram Descriptor) for feature extraction.

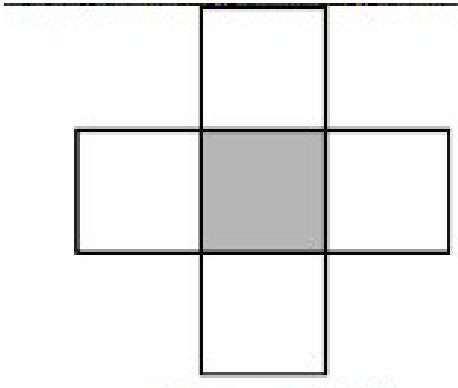

Square grid (4)

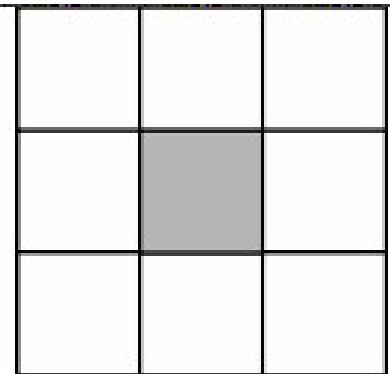

Square grid (8)

FIG. 6(a). 2D STRUCTURING ELEMENTS

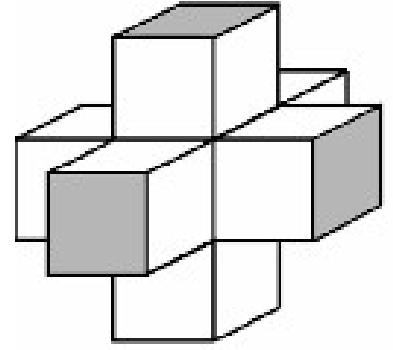

6 voxels

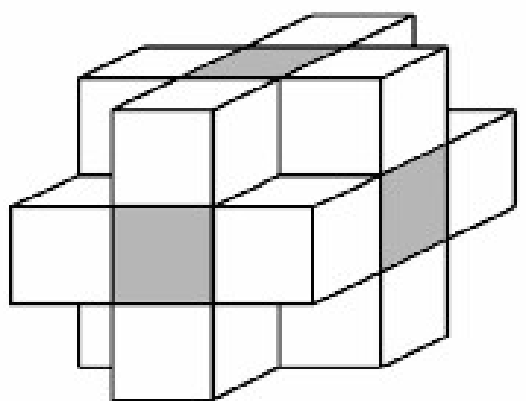

18 voxels

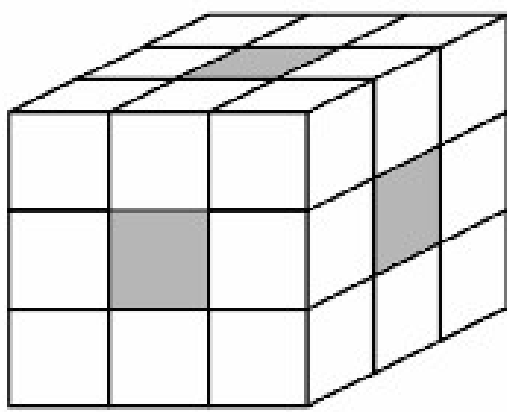

26 voxels

FIG. 6(b). 3D STRUCTURING ELEMENTS

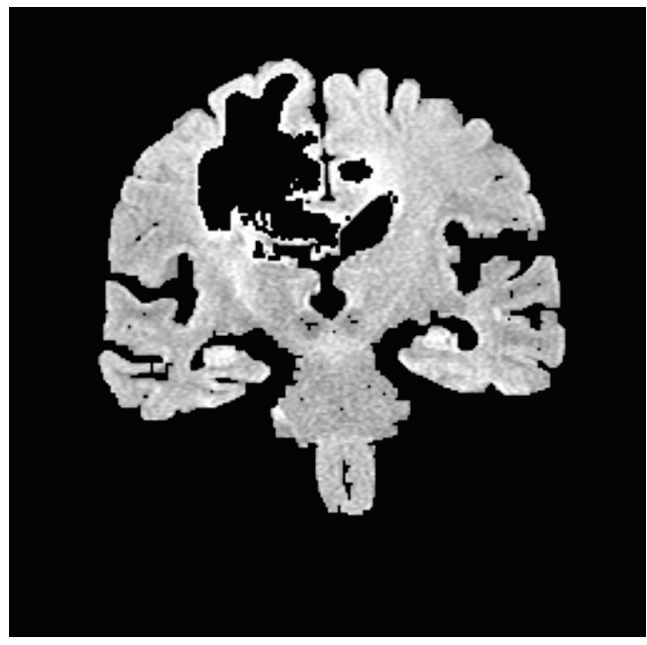

FIG. 7(a). MORPHOLOGICAL ERODED BRAIN

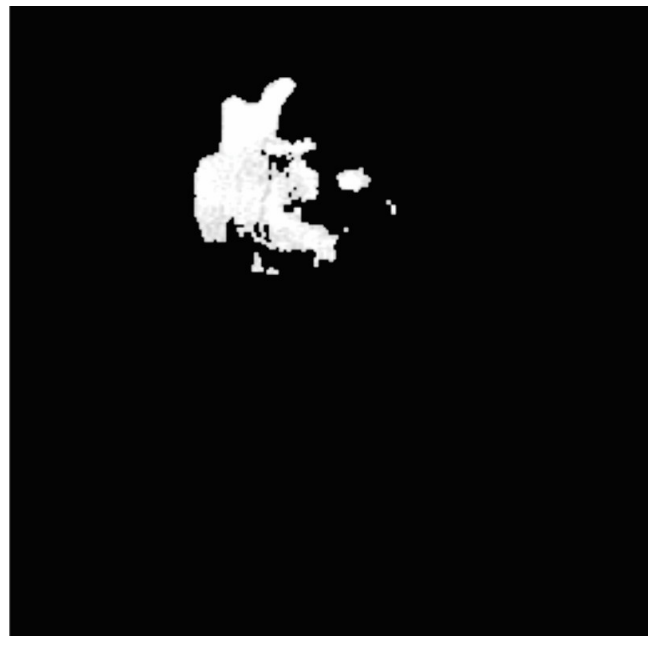

FIG. 7(b). MORPHOLOGICAL ERODED TUMOR

Mehran University Research Journal of Engineering \& Technology, Volume 38, No. 3, July, 2019 [p-ISSN: 0254-7821, e-ISSN: 2413-7219] 
EHD descriptor compute the direction of edges in different angles. In the first phase of EHD descriptor, the image is divided into 16 non-overlapping blocks of the same size. Each image block is then split into four sub-blocks and assigned the labels from 0-3. Average gray level for each block is then calculated as $a_{k 0}(i, j)$. For each direction, filter co-efficient is represented as $f$. Each of the image blocks is then classified into one of the five mentioned edge categories or as a non-directional edge block. Now, the magnitude is represented as $m$. The proposed framework is shown in Figs. 8-9(a-e).

$$
\begin{aligned}
& \mathrm{m}_{\mathrm{v} 0}(\mathrm{i}, \mathrm{j})=\left|0 \sum_{\mathrm{k}=00}^{30} a_{\mathrm{k} 0}(\mathrm{I}, \mathrm{j}) \times \mathrm{f}_{\mathrm{v} 0}(\mathrm{k})\right| \\
& \mathrm{m}_{\mathrm{k} 0}(\mathrm{i}, \mathrm{j})=\left|\sum_{\mathrm{k}=00}^{30} a_{\mathrm{k} 0}(\mathrm{I}, \mathrm{j}) \times \mathrm{f}_{\mathrm{k} 0}(\mathrm{k})\right| \\
& \mathrm{m}_{\mathrm{d}-450}(\mathrm{i}, \mathrm{j})=\left|\sum_{\mathrm{k}=00}^{30} a_{\mathrm{k} 0}(\mathrm{I}, \mathrm{j}) \times \mathrm{f}_{\mathrm{d}-450}(\mathrm{k})\right| \\
& \mathrm{m}_{\mathrm{d}-1350}(\mathrm{i}, \mathrm{j})=\left|\sum_{\mathrm{k}=00}^{30} a_{\mathrm{k} 0}(\mathrm{I}, \mathrm{j}) \times \mathrm{f}_{\mathrm{d}-1350}(\mathrm{k})\right|
\end{aligned}
$$

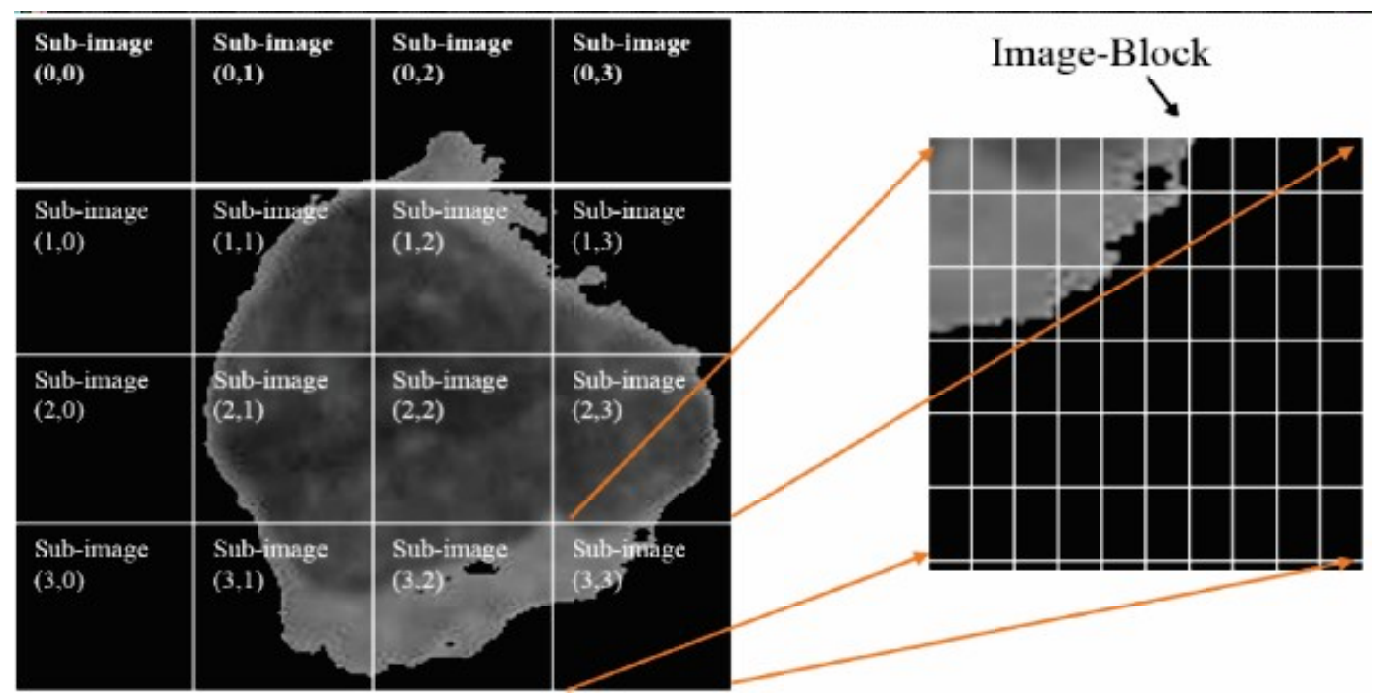

FIG. 8. DEFINITION OF SUB IMAGE AND IMAGE BLOCK
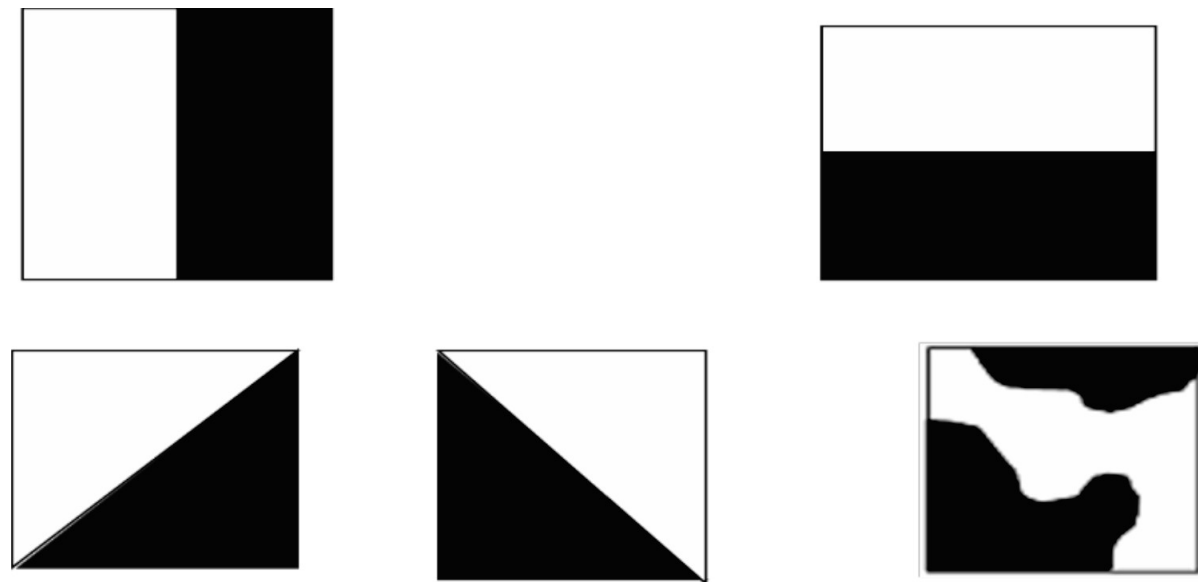

FIG. 9. FIVE TYPES OF EDGES

Mehran University Research Journal of Engineering \& Technology, Volume 38, No. 3, July, 2019 [p-ISSN: 0254-7821, e-ISSN: 2413-7219] 
$\mathrm{m}_{\mathrm{nd} 0}(\mathrm{i}, \mathrm{j})=\left|\sum_{\mathrm{k}=00}^{30} a_{\mathrm{k} 0}(\mathrm{I}, \mathrm{j}) \times \mathrm{f}_{\mathrm{nd} 0}(\mathrm{k})\right|$

Equations (1-5) are derived from Figs. 8-9. where $\mathrm{m}$ is magnitude, $v$ is vertical direction, $k$ is the location of sub blocks, $d$ is the directional, $n d$ is the non-directional, $\Sigma$ is sigma, $a$ is the average, $f$ is the filter coefficient and $(I, j)$ specifies the location of image block. Mostly we deal with the five types of edges therefore, we use five clicks. A total number of features extracted from 16 blocks are 80 numerical values whose data type is floating point, where every feature is actually the frequency of the edges in a certain block of image histogram as shown in Fig. 10 and Table 1.

\subsection{Classification}

SVM classifier is used for classification, Supervised Classifier is trained on the edges angles features extracted from the ROI (benign and Malignant). The proposed classification framework is shown in Fig. 11. Then the classifier finds out the difference between benign and malignant features. Similarly, a class (benign or malignant) is assigned by the SVM classifier to the new brain tumor test image. State-of-art machine learning SVM classifier is used to diagnose two classes of cancer (i.e. benign and malignant). The kernel in classifiers is used to remove the outlier located in the wrong class which affects the precision of the classifier. In our work, we used geometrical features which are very robust and do not contain any outliers. SVM draws hyperplane which can be linear or non-linear. Many hyperplanes can be drawn in case of two classes but the optimal one is selected which separates the features of two classes with maximum margins. Hyperplane is a line (i.e. straight or curly). It, in our case, can be welldefined in Equations (6-9):

$x_{0}+0_{b}=00$

TABLE 1. SEMANTICS OF LOCAL EDGE

\begin{tabular}{|c|c|}
\hline Histogram Bins & Semantics \\
\hline BinCount(0) & Straight line Vertical edges \\
\hline BinCount(1) & Straight line Horizontal edges \\
\hline BinCount(2) & Diagonal 450-degree edges \\
\hline BinCount(3) & Diagonal 1350-degree edges \\
\hline BinCount(4) & Non-directional curly edges \\
\hline BinCount(5) & Straight line Vertical edges \\
\hline BinCount(79) & \\
\hline BinCount(75) & Straight line Vertical edges \\
\hline BinCount(76) & Straight line Horizontal edges \\
\hline BinCount(77) & Diagonal 450-degree edges \\
\hline & \\
\hline Bincount(78) 1350-degree edges \\
\hline
\end{tabular}
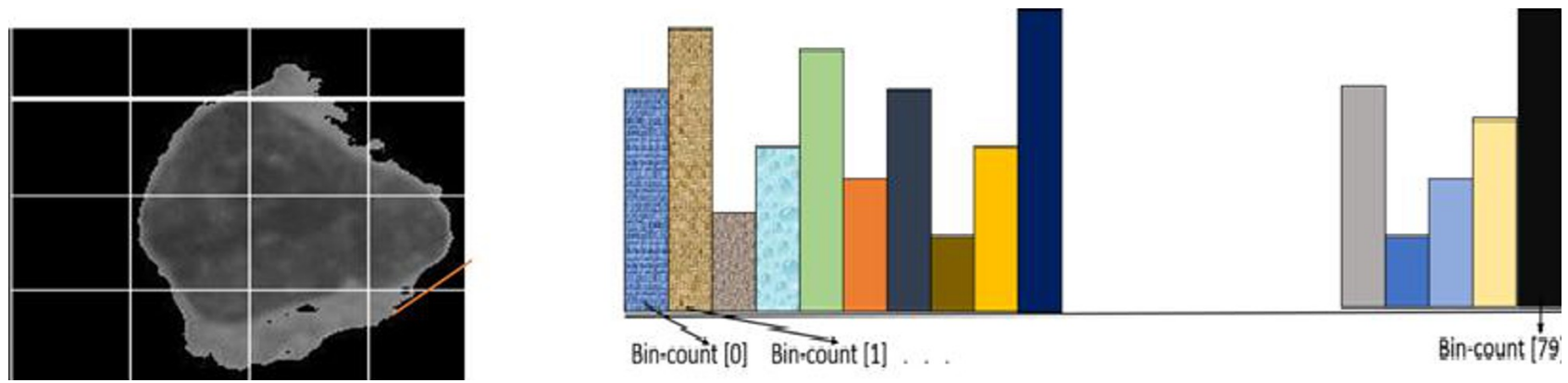

FIG 10. ONE DIMENSIONAL ARRAY OF 80 BINS OF EHD

Mehran University Research Journal of Engineering \& Technology, Volume 38, No. 3, July, 2019 [p-ISSN: 0254-7821, e-ISSN: 2413-7219] 
Where $x$ and $y$ can be defined as points on hyperplane, similarly, b defined as bias and w defined as the mean/ average of the Euclidean.

$w x_{i 0}+\mathrm{b} 0 \geq 0+1,0 \mathrm{y}_{\mathrm{i}}=+10$

$w x_{i 0}+\mathrm{b} \leq 0-1, \mathrm{y}_{\mathrm{i}}=0-1$

$y_{i 0}\left(w x_{i 0}+\mathrm{b}\right)-1 \geq 00$

\subsection{Evaluation of the Proposed Segmentation Method}

A subjective performance evaluation of the proposed segmentation method is performed using sensitivity, specificity, FNR (False Negative Rate), FPR (False Positive Rate), and F-measure as shown in Equations (10-14):

Sensitivity $=\frac{\mathrm{TP}}{\mathrm{TP}+\mathrm{FN}}$

Specificity $=\frac{\mathrm{TN}}{\mathrm{TN}+\mathrm{FN}}$

$\mathrm{FNR}=\frac{\mathrm{FN}}{\mathrm{TP}+\mathrm{FN}}$
$\mathrm{FPR}=\frac{\mathrm{FP}}{\mathrm{TN}+\mathrm{FN}}$

$\mathrm{F}-$ Measure $=2 \times \frac{\mathrm{FPR} * \mathrm{FNR}}{\mathrm{FPR}+\mathrm{FNR}}$

where TP is defined as the True Positive, FN as False Negative, similarly TN as True Negative and FP as False Positive.

\subsection{Dataset and Tool}

The Dataset for the proposed method is obtained from the radiology department of Shifa International Hospital Ltd. Faisalabad, Pakistan. Images were acquired through the open 35Tesla MRI machine. The whole proposed work is implemented by using MATLAB.

\section{EXPERIMENTAL WORK}

Detailed experimental work is carried out to check the efficiency and robustness of the planned segmentation and classification methods. Experimental work has two parts i.e. segmentation and classification which are discussed in Tables 2-3 and Figs. 11-12. The graphical comparison is shown in Figs. 13-15.

TABLE 2. TUMOR SEGMENTATION METHOD COMPARISON WITH SOME STATE-OF-THE-ART METHODS

\begin{tabular}{|c|c|c|c|c|c|}
\hline Method & Sensitiveness & Preciseness & FPR & FNR & F-Measure \\
\hline Ray [19] & 0.99900 & 0.98200 & 0.11500 & 0.67500 & 0.8900 \\
\hline Irfan et. al. [28] & 0.98200 & 0.99100 & 0.06900 & 0.17800 & 0.8800 \\
\hline Haiyan et. al. [27] & 0.97300 & 0.99300 & 0.0500 & 0.09900 & 0.9600 \\
\hline Proposed Method & 0.94100 & 0.98100 & 0.04900 & 0.098800 & 0.9700 \\
\hline \multicolumn{7}{|c|}{ *Values in Brackets Represent the SD } \\
\hline
\end{tabular}

TABLE 3. PERFORMANCE COMPARISON WITH SOME BENCHMARK CLASSIFIERS

\begin{tabular}{|c|c|c|c|c|}
\hline $\begin{array}{c}\text { Classification } \\
\text { Approach }\end{array}$ & $\begin{array}{c}\text { Specificity } \\
(\%)\end{array}$ & $\begin{array}{c}\text { Sensitivity } \\
(\%)\end{array}$ & $\begin{array}{c}\text { Precision } \\
(\%)\end{array}$ & $\begin{array}{c}\text { Mean Square } \\
\text { Error }\end{array}$ \\
\hline $\begin{array}{c}\text { Backpropagation Neural } \\
\text { Network }\end{array}$ & 68.1700 & 89.5800 & 88.8500 & 0.2100 \\
\hline K-Nearest Neighbors & 76.1900 & 91.8400 & 91.1400 & 0.1000 \\
\hline Fusion RGSA & 95.000 & 98.9400 & 98.400 & 0.01500 \\
\hline Projected & 97.6100 & 99.7100 & 99.0100 & 0.09000 \\
\hline
\end{tabular}

Mehran University Research Journal of Engineering \& Technology, Volume 38, No. 3, July, 2019 [p-ISSN: 0254-7821, e-ISSN: 2413-7219] 


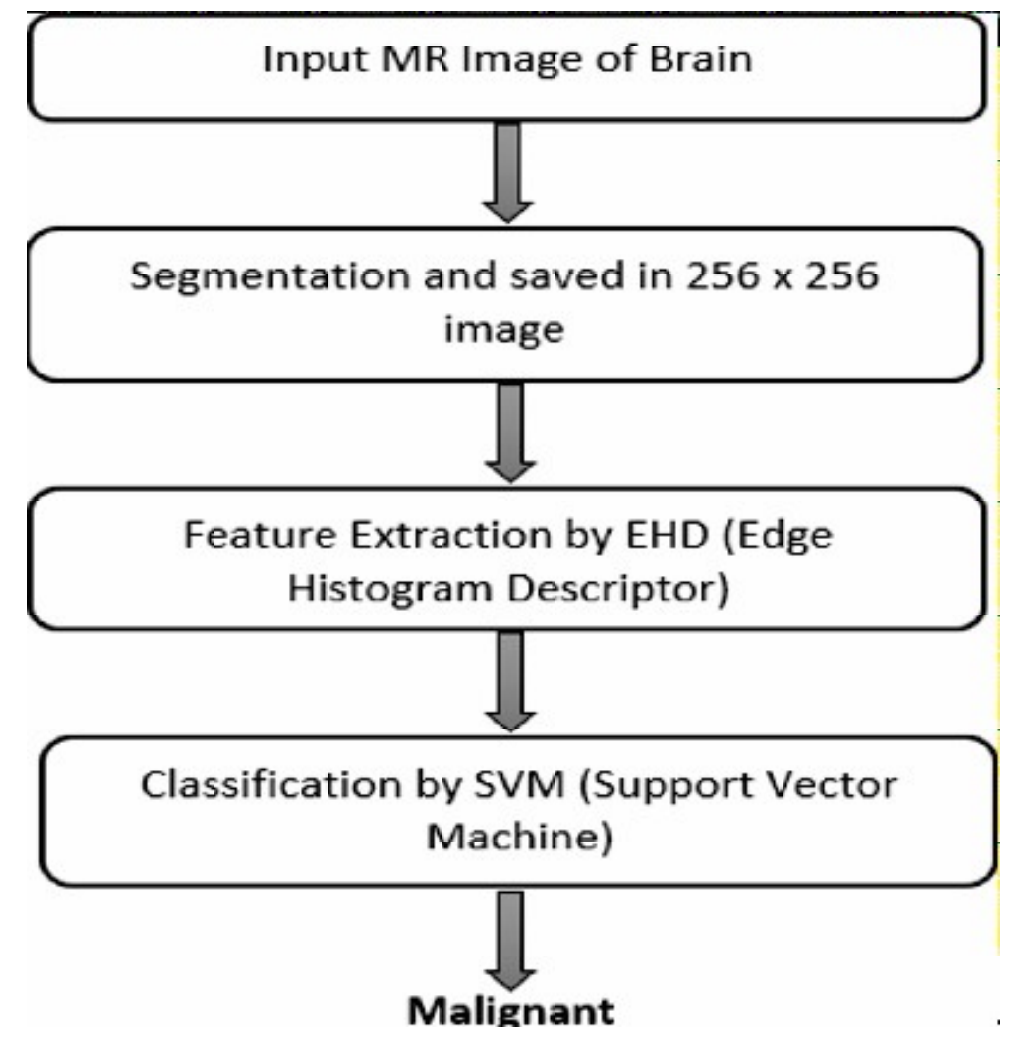

FIG. 11. THE PROPOSED FRAMEWORK FOR CLASSIFICATION

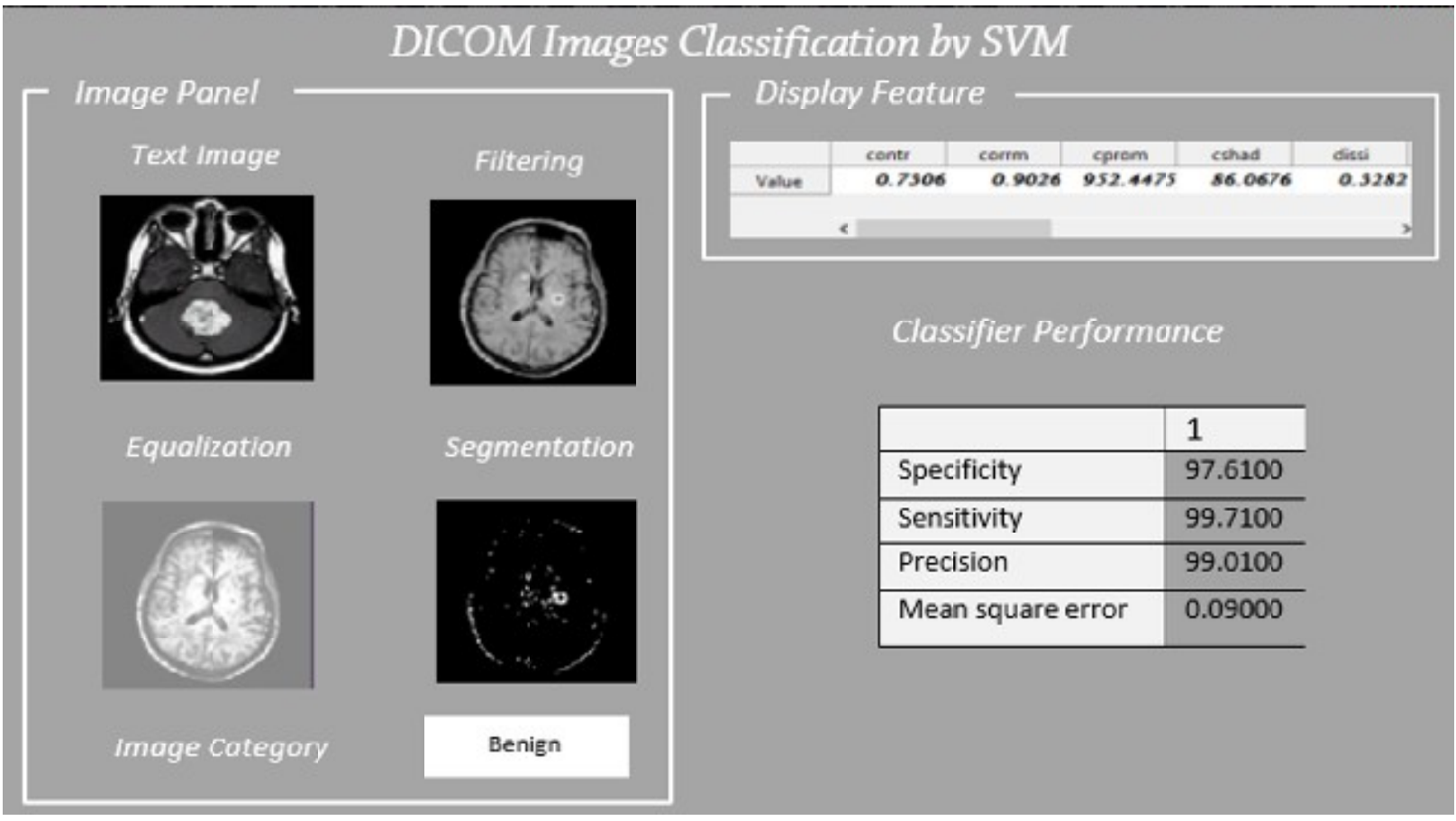

FIG. 12. CLASSIFIED OUTPUT

Mehran University Research Journal of Engineering \& Technology, Volume 38, No. 3, July, 2019 [p-ISSN: 0254-7821, e-ISSN: 2413-7219] 
Brain MRI
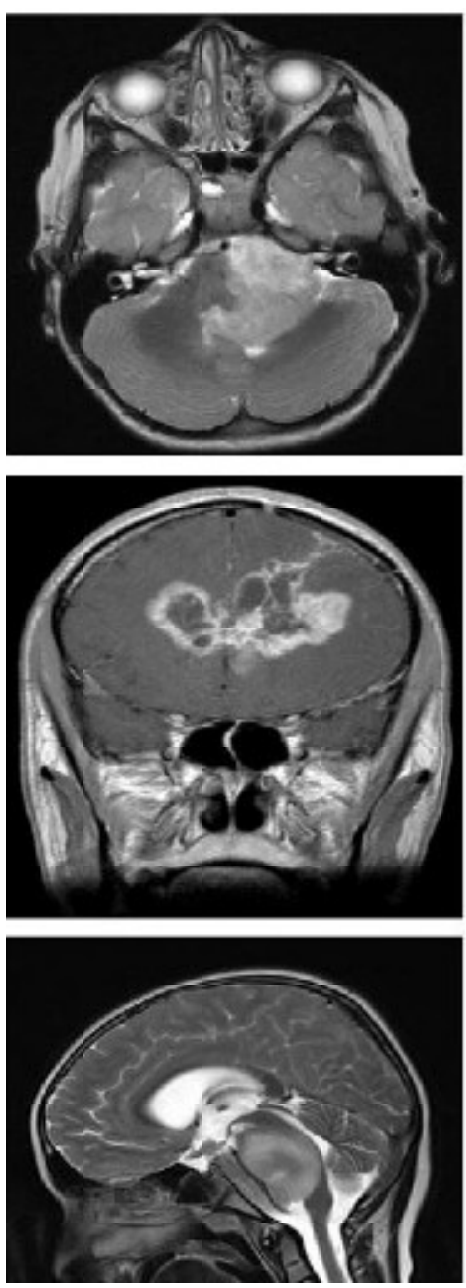

Segmented Tumor
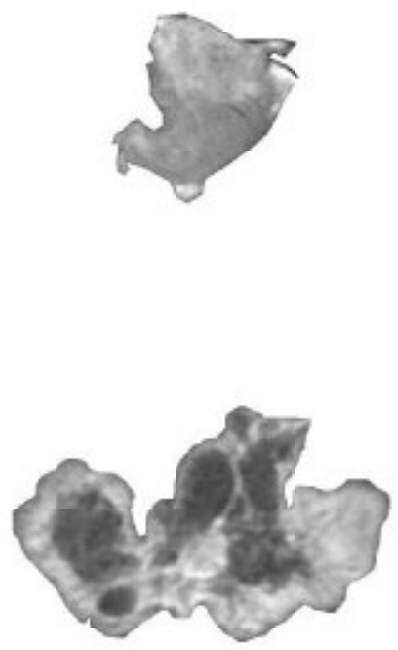

FIG. 13. BRAIN TUMOR MRI AND SEGMENTED TUMOR REGIONS

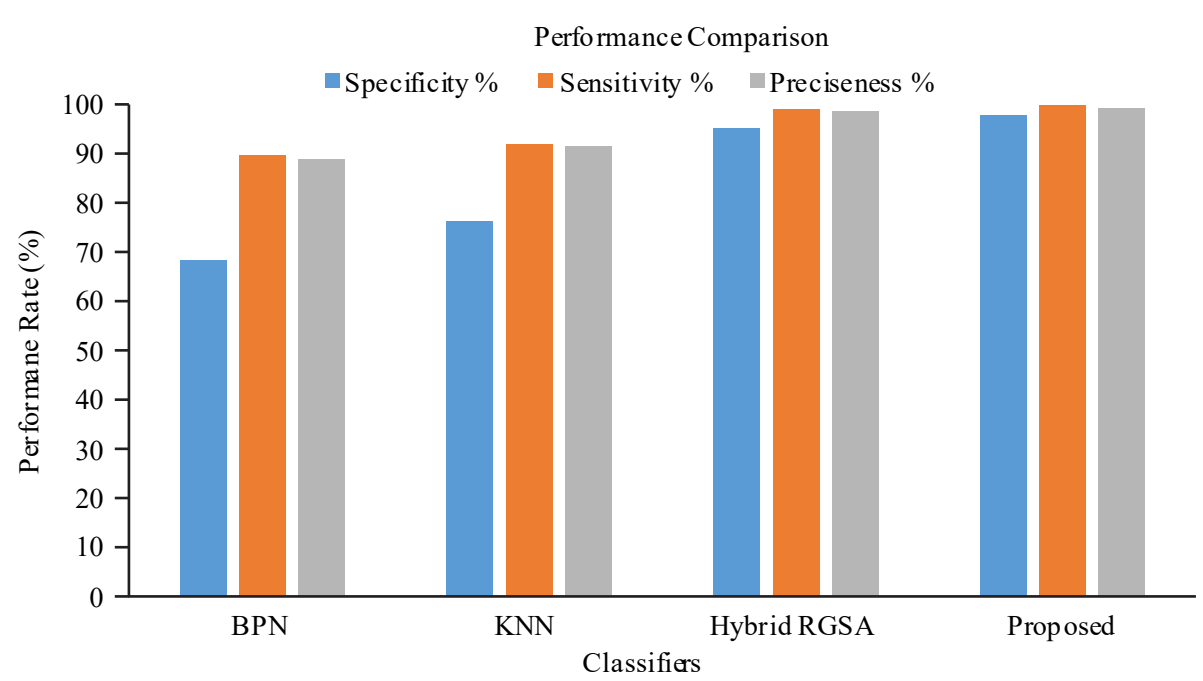

FIG. 14. EVALUATION OF OUR PROJECTED CLASSIFIER WITH SOME STATE-OF-THE-ART CLASSIFIERS

Mehran University Research Journal of Engineering \& Technology, Volume 38, No. 3, July, 2019 [p-ISSN: 0254-7821, e-ISSN: 2413-7219] 


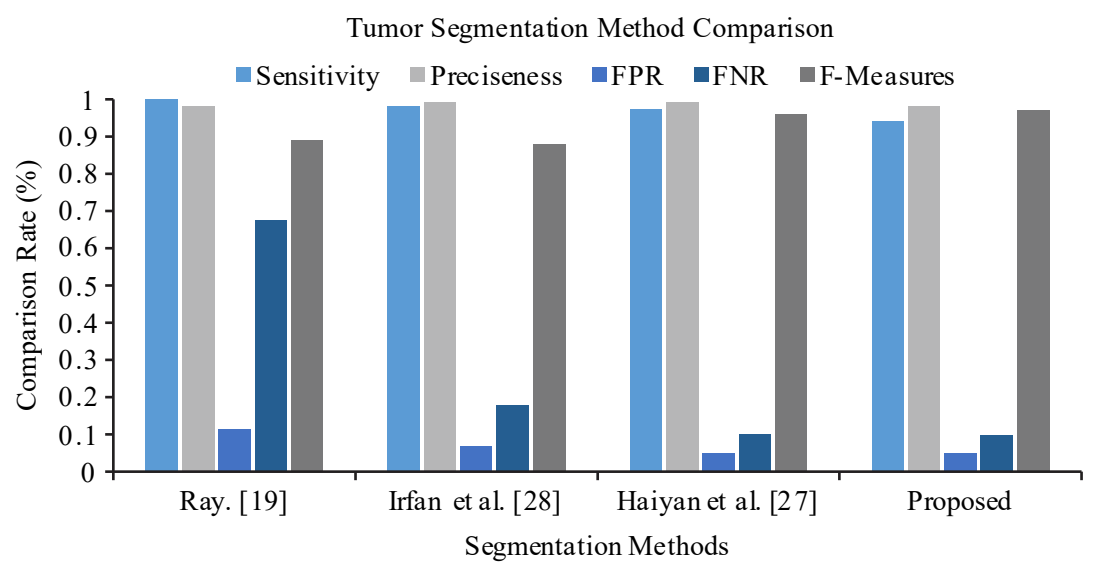

FIG. 15. PROJECTED SEGMENTATION METHOD COMPARISON WITH SOME STATE-OF-THE-ART METHODS

\section{CONCLUSION}

In this paper, authors have presented a framework which is very efficient for the diagnosis of tumors in brain MRI DICOM images. First of all, the tumor is segmented efficiently from the MRI slices using 3D segmentation technique which automatically select an optimum threshold value for brain and cancer voxels. SVM classifier with BOVW features is used which achieves higher accuracy for classifying two class of tumors (Malignant and benign). The proposed framework performs important diagnosis tasks in biomedical imaging. Experimental study validates the efficiency of the proposed system.

\section{ACKNOWLEDGEMENT}

This research work was supported and conducted with the help of the Department of Information Technology, Government College University, Faisalabad, Pakistan.

\section{REFERENCES}

[1] Zhang, Y.Z., Yuan, T.F., and Dong, Z.C., "Brain Imaging and Automatic Analysis in Neurological and Psychiatric Diseases..Part- I”, CNS \& Neurological Disorders-Drug Targets (Formerly Current Drug Targets-CNS \& Neurological Disorders), Volume 16, pp. 3-4, 2017.
[2] Enhancement, B.I., "Guest Editorial: Medical Image Computing at the Institute of Mathematics and Computer Science in Medicine, University Hospital Hamburg - Eppendorf", Volume 21, No. 7, pp. 713-723, 2002.

[3] Goossens, I.D.T.B., and Philips, W., "MRI Segmentation of the Human Brain: Challenges, Methods, and Applications", Computational and Mathematical Methods in Medicine, pp 1-23, 2015.

[4] Natarajan, P., Krishnan, N., Kenkre, N.S., Nancy, S., and Singh, B.P., "Tumor Detection using Threshold Operation in MRI Brain Images”, Proceedings of IEEE International Conference on Computational Intelligence \& Computing Research, pp. 1-4, 2012.

[5] Algohary, A.O., El-bialy, A.M., Kandil, A.H., and Osman, N.F., "Improved Segmentation Technique to Detect Cardiac Infarction in MRI C-SENC Images", Proceedings of $5^{\text {th }}$ International Conference on Biomedical Engineering, pp. 21-24, Cairo, December, 2010.

[6] Viji, K.S.A., "Automatic Detection of Brain Tumor based on Magnetic Resonance Image using CAD System with Watershed Segmentation", Procedings of International Conference in Signal Processing, Communication, Computing and Networking Technologies, pp. 145-150, 2011.

[7] Das, A.J., Mahanta, L.B., and Prasad, V., “Automatic Detection of Brain Tumor from MR Images Using Morphological Operations and K-Means Based Segmentation", Proceedings of Conference on Emerging Research in Computing, Information, Communication and Applications, Volume 2, Bangalore, 2016. 
[8] Ahmad, A., and Dey, L., “A K-Mean Clustering Algorithm for Mixed Numeric and Categorical Data", Data Knowledge Engineering, Volume 63, No. 2, pp. $503-527,2007$.

Deng, W., Xiao, W., Deng, H., and Liu, J., "MRI Brain Tumor Segmentation With Region Growing Method Based on the Gradients and Variances along and Inside of the Boundary Curve", Proceedings of $3^{\text {rd }}$ International Conference In Biomedical Engineering and Informatics, Volume 1, pp. 393-396, 2010.

[10] Khotanlou, H., Colliot, O., Atif, J., and Bloch, I., “3D Brain Tumor Segmentation in MRI Using Fuzzy Classification, Symmetry Analysis, and Spatially Constrained Deformable Models”, Fuzzy Sets System, Volume 160, No. 10, pp. 1457-1473, 2009.

[11] Ghosh, S., "Comparative Analysis of K-Means and Fuzzy C-Means Algorithms", International Journal of Advanced Computer Science and Applications, Volume 4, No. 4, pp. 35-39, 2013.

[12] Yazdani, S., Yusof, R., Riazi, A., and Karimian, A., "Magnetic Resonance Image Tissue Classification Using an Automatic Method", Diagnostic Pathology, Volume 9, pp. 1-16, 2014.

[13] Kong, H., Dqg, K., Dvx, Q.X.S., Ri, H., Oehuwd, R.R.I., Phwkrg, V., Rq, E., Dxvvldq, D.H.G., Rq, P., Lv, Y., Dydlodeoh, D.S., Eudlq, Q.G.H., Vhjphqwdwlrq, W., and Lorz, Y., "Fully Automatic Brain Tumor Segmentation Using a Normalized Gaussian Bayesian Classifier and 3D Fluid Vector Flow", Proceedings of IEEE 17th International Conference on Image Processing, pp. 2553-2556, 2010 .

[14] Juan-Albarracín, J., Fuster-Garcia, E., and Manjón, J.V., "Automated Glioblastoma Segmentation Based on a Multiparametric Structured Unsupervised Classification", PloS One, Volume 10, No. 5, e0125143, 2015.

[15] Zakeri, F.S., and Behnam, H., "Classification of Benign and Malignant Breast Masses Based on Shape and Texture Features in Sonography Images", Journal of Medical Systems, Volume 36, No. 3, pp. 1621-1627, 2012.
[16] Galen, Y., Yudong, Z., Jiquan, Y., Genlin, J., Dong, Z., Wang, S., Feng, C., and Wang, Q., "Automated Classification of Brain Images Using Wavelet-Energy and Biogeography-Based Optimization", Multimedia Tools and Applications, Volume 75.23, pp. 15601-15617, 2016.

[17] Zhang, Y.D., Yuan, T.F., and Dong, Z., "Brain Imaging and Automatic Analysis in Neurological and Psychiatric Diseases Part-II", CNS \& Neurological Disorders-Drug Targets (Formerly Current Drug Targets-CNS \& Neurological Disorders), Volume 16, No. 2, pp. 114-115, 2017.

[18] Mehmood I., Sajjad M., Muhammad, K., and Syed, I., "An Efficient Computerized Decision Support System for the Analysis and 3D Visualization of Brain Tumor", Multimedia Tools and Applications, pp. 1-26, 2018.

[19] Ray, D., "Noise Reduction and Image Enhancement of MRI using Adaptive Multiscale Data Condensation", Proceedings of 1st International Conference In Recent Advances in Information Technology, pp. 107-113, 2012.

[20] Bagheri, A.M., Montazer, G.A., and Escalera, S., "ErrorCorrecting Output Codes for Multiclass Classification: Application to Two Image Vision Problems", $16^{\text {th }}$ International Symposium on Artificial Intelligence and Signal Processing, pp. 508-513, 2012.

[21] http://www.labbookpages.co.uk/software/imgProc/ otsuThreshold.html, (Accessed: 20 ${ }^{\text {th }}$ August, 2018 at GMT 10:40 pm).

[22] Zhang, H., Liu, J., Zhu, Z., and Li, H., “An Automated and Simple Method for Brain MR Image Extraction", Biomed. Engineering, Online, Volume 10, No. 1, pp. 81, 2011.

[23] Har-Peled, S., and Roth, D., "Constraint Classification for Multiclass Classification and Ranking”, Urbana, Volume 51, pp. 61801, 2002.

[24] El-Dahshan, E.S.A., Hosny, T., and Salem, A.B.M., "Hybrid Intelligent Techniques for MRI Brain Images Classification”, Digital Signal Processing, A Review, Journal, Volume 20, No. 2, pp. 433-441, 2010. 
[25] Sharma, R.R., and Marikkannu, P., "Hybrid RGSA and Support Vector Machine Framework for ThreeDimensional Magnetic Resonance Brain Tumor Classification”, The Scientific World Journal, 2015.

[26] Jaffar, M.A., Zia, S., Latif, G., and Mirza, A., “Anisotropic Diffusion-Based Brain MRI Segmentation and 3D Reconstruction", International Journal of Computational Intelligence Systems, Volume 5, No. 3, pp. 494-504, 2012 .

[27] Haiyan, Z., Jiafeng, L., Zixin, Z., and Haiyun, L., “An Automated and Simple Method for Brain MR Image Extraction", Biomedical Engineering, Online, Volume 10 No. 1, pp. 81, 2011.
[28] Irfan, M., Naveed, E., Sajjad, M., and Baik, S.W., "Prioritization of Brain MRI Volumes Using Medical Image Perception Model and Tumor Region Segmentation", Computers in Biology and Medicine, Volume 43, No. 10, pp. 1471-1483, 2013.

[29] Irfan, M., Sajjad, M., and Baik, S.W., "Mobile-Cloud Assisted Video Summarization Framework for Efficient Management of Remote Sensing Data Generated by Wireless Capsule Sensors", Sensors, Volume 14, No. 9, pp. 17112-17145, 2017.

[30] https://www.lrh.gov.pk/, (Accessed: 10 $10^{\text {th }}$ July, 2018 at GMT 11:40 pm). 